\section{Underpinnings of Medical Ethics}

\author{
Edmond A Murphy, James J Butzow \\ and Edward L Suarez-Murias, \\ Baltimore and London, The Johns \\ Hopkins University Press, 1997, \\ $\mathrm{xv}+480$ pages, $£ 41.50 \mathrm{hc}$.
}

This book represents an opportunity lost. The authors are respectively an emeritus professor of medicine and former head of medical genetics at The Johns Hopkins School of Medicine; a retired research biochemist in the Laboratory of Cellular and Molecular Biology, National Institute on Aging; and a psychiatrist in private practice who is also an assistant professor of psychiatry at Johns Hopkins. Their background is therefore medical rather than philosophical, and they claim in their preface that their "exclusive interest in the book is with safeguarding the supply of Medical knowledge as raw material to be delivered to the Medical ethicist" and that their goal is "to explore some of the major areas of confusion that exist in the no-man's-land between scholarly clinical Medicine and the professional ethicist, typically nurtured in Philosophy". They present their project with some assertiveness: "We do not want Medicine to be cast in the role of a poor relation or of a Third World nation to be colonized by experts from advanced civilisations bartering raw clinical materials at a trading post of academic law, or Philosophy, or theology' (page 5). (The eccentric capitalisation is the authors' own - see below.)

In the light of these remarks I hoped for two things: an formal counterpart, with examples, of the claims that one often hears in an informal way from health care workers - that philosophers, lawyers and theologians tend to be confused or ignorant about what actually goes on in the areas of medicine that they pronounce on so confidently; and an attempt to provide a more adequate factual "underpinning", to use their own word, for the ethicists' activities in some crucial areas. Instead the book is often more like a study in the philosophy of the scientific aspects of medicine, and the relevance to ethics is sometimes remote.

Perhaps one reason behind this defect is that the authors are seriously unclear about what they mean by "ethics". For example, on page 20 "Major Ethics" is described as "the study of the relationship between Acts and their consequences", "Minor Ethics" as the application of means to broad classes of ends. In several places ethical statements are characterised as descriptive, rather than optative or imperative. One might conclude from these points that the authors are (eccentrically) using the term "medical ethics" for the study of how various desirable goals in the health field are as a matter of fact achieved. Moreover, in this book Ethics is normally contrasted with Morals (itself ambiguously characterised, as "what pertains to the role of conscience" on page xiv but as "norms of behaviour derived from authority" on page 17) which seems to be about what one ought to do. The book claims not to deal with morals. However, there is a chapter called "Ethical Systems" which takes "ethics" in its normal meaning and is, as one would expect, about theories of what ought to be done, whether in medicine or in life in general.

The chapter called "Duration and Quality of Life" provides a good example of how the approach fails to connect with medical ethics as normally understood. Most people would expect a discussion which, whether or not it mentioned QALYs (quality adjusted life years), had a bearing on the kind of ethical issue to which duration and quality of life apply. But this chapter begins with long discussions of what it is for a cell to be alive, the proper account of an individual (organism), three ways in which complex organisms can be studied, various ways in which the survivorship of a cohort can be diagrammatically portrayed, and so on. The points made about the obscurity of some claims about life expectancy presumably have ethical relevance (for example to the comparative evaluation of alternative treatment policies), but this is not made clear. What is finally said about quality of life is fairly perfunctory and tends to suggest that the chief measure of it is the satisfaction of the owner of the life and those who come in contact with him or her. But it is not clear how this view relates to the earlier examples of blind and deaf geniuses: their quality of life does not obviously depend on their being happy. The authors understandably profess a reluctance to prescribe what quality of life is, but again a fuller account of possible views and the ethical issues those views might raise would have been welcome. The last chapter, "The Main Perspectives", is an even clearer example of the problems of this book; I hoped for a final summary of what had be shown, but the chapter peters out in a discussion of different senses of "function" found in evolutionary bic ogy.

There is a good deal of incident interest in this book. But I cannot re ommend it as a whole as a contrib tion to medical ethics. And the ince dental merits of it are often obscuref by a tiresomely long-winded and inflated style. A fairly typical examp.R (in the final discussion of function "The word fitness is floridly [s] ambiguous. But it is neither courteo离 nor enlightening to harp on the amb guity to the exclusion of all else. In cannot peremptorily suppose that aلl using the term are fools who do no look where they are going" (page 396 And the aforementioned capitalisation system "to denote a special technic sense" is well-meaning, but in the end - or somewhat sooner - it too become tiresome. In my view it is als unnecessary. Perhaps in some con texts, though not in this book, we need to distinguish the Philosopher ( $\vec{\Phi}$ scholar who studies values and redif by speculative rather than empigial means") from the philosopher benign contemplative person of table temperament"); but ambiguity unlikely between medicine ("a ther peutic concoction") and Medicir ("the professional care of health an disease among human beings").

\section{ELIZABETH TELFE Deparment of Philosoph University of Glasg}

\section{The Powerful Placebo}

A K Shapiro and E Shapiro,

Baltimore and London, The Johns Hopkins University Press, 1997, 290 pages, 233 .

The book kicks off with an historic account of medicine which fills up thro best part of the first chapter. We ses్ how medicines have been modified tweaked or superseded throughout the ages, from the simple "laying on hands" to the more bizarre concoc tions such as dirt laced with flyspecks and lizard's blood (taken from thw Egyptian Ebers Papyrus, circa $150 \widehat{\widehat{\Phi}}$ $\mathrm{BC})$ or procedures such as subjectin $\vec{\Phi}$ patients to a human centrifuge. Th narrative is highly detailed and probe ably exhaustive, only to conclude wite the speculation (and speculation it is) that most, if not all, treatments before the twentieth century at least can be 
reduced to placebos. Lots of primitive treatments seem to have had real negative effects, however, as the authors later point out.

The ensuing discussion of semantics, which is again somewhat detailed but interesting nevertheless, culminates in a conceptual schema. The authors propose that the etymology of the term "placebo" should come full circle and that its definition should not be restricted to the relatively recent "inert substance" of the twentieth century. Rather, it should include active and inert substances, as well as physiological and psychological treatments. The definition of a placebo is anything that has a placebo effect. So far so good. A placebo effect is any effect that is not specific, so a placebo can be given knowingly or not. All therapies have effects that are part placebo, part non-placebo, the ratio of which the authors illustrate by the fact that predictable and specific effects increase with higher dosages. The example given is the anti-psychotic drug chlorpromazine which has a high placebo to non-placebo ratio at low, homoeopathic dosages, and a low placebo to non-placebo ratio at high dosages, indicating that the most predictable and highly specific effects occur at massive overdoses. However, the reader is left unsure as to what counts as a specific effect. Without knowledge of how a treatment works, it is difficult to know whether its effects are specific or not: perhaps doing a double-blind study would provide the answer, but then the definition may be circular. Or perhaps we could look at what effects are predicable, an approach that is implicit in the authors' discussion, but this rests on the erroneous assumption that predictability implies specificity. Massive overdoses may well lead predictably to death, but this not specific to any one disease. Mortality is a specific outcome measure, granted, but it is not the only outcome. High dosages of a drug could result in a wholesale shut-down of organs, or could at least have multiple if not fatal effects. Take aspirin for example, whose various analgesic and prophylactic effects have been well documented. Nor does specificity imply predictability. Even if we were to agree on a specific outcome, it might be difficult to predict the effects on individuals in light of population data. The definition as it stands runs the risk of being so broad as to be more or less wide open, so it is small wonder that the authors view much of prescientific therapy as placebo. The authors seem to want too much from their definition, conceding in the end that it may prove too inclusive. On the bright side, contributory factors to the placebo effect such as the natural course of the illness in question, regression to the mean, clinical bias, and methodological variables are excluded from the schema.

Quite rightly, the authors show how the introduction of scientific method to medicine and psychology reserved a respectable place for the placebo under single- and double-blind methods to evaluate therapeutics in controlled clinical trials. By controlling for the influence of patients' expectations (using single-blind studies) with additional control for observer bias (using double-blind ones), any treatment effect can be attributed to the specific pharmacological or psychological activity of the treatment in question, according to the Shapiro/Shapiro schema.

The advance of randomisation to avoid selection bias is given only brief mention. Later on in the book, the authors ask how blind is blind and provide a review of published literature plus some primary data of their own to evaluate the extent to which physicians and patients, among others, are able to guess their treatment assignment. Only tentative inferences can be drawn from the data, as the authors point out, but they still recommend the use of "matching placebos" which are identical to the experimental treatment, at least with respect to physical characteristics. There is very little in the way of discussion on the possible scientific value of "open" (non-blind) trials. By contrast, there is a good account of the relative scientific merits of placebocontrolled trials and comparative trials of two or more active agents.

There is limited analysis of the ethical issues raised by the use of placebo in practice and in research particularly. The analysis is limited both in terms of scope and depth. With regard to the former, sham operations are barely mentioned and issues such as placebo-controlled trials in Third World countries when a "proven" treatment already exists are not covered, though the authors do talk of "uncertainty" between trial arms being a moral prerequisite of trials and the unacceptability of withholding "proven" and freely available treatments from control patients. What if a country cannot afford the "proven" treatment, would it be ethical to sanction a placebo-controlled trial of a new therapy? Nor are ideas of uncertainty or, less ambiguously, equipoise taken further, which is unfortunate since the authors claim that some of the ethical constraints can be circumvented by variations in experimental design. This may indeed be so, though not necessarily in the way the authors intend. When a patient's status worsens or the patient fails to improve or develops adverse effects, the authors suggest that the clinician might remove the patient from the trial or administer an active therapy. Is this not standard procedure? In any case, the possibility that such withdrawals may compromise the scientific value of the study by introducing bias (depending on what outcome measures are used) or weaken any association, are not covered. Another difficulty, arising out of an inadequate discussion of equipoise and no mention of decision analysis, is the claim that placebo control patients may be subject to less risk than their experimental counterparts. Given genuine uncertainty (equipoise in a decision analytic framework) this may be the case, but there would be the chance of benefit for experimental patients sufficient to offset such risks. Particularly relevant here is the use of sham operations where risks may not be balanced by expected benefits. Also, the ethical implications of using "active" placebo drugs that mimic adverse effects of active treatments in an attempt to maintain the integrity of the doubleblind without promising any specific therapeutic benefit are not brought out.

SARAH J L EDWARDS

Department of Public Health and Epidemiology University of Birmingham

\section{Advance Directives or Living Wills}

\section{The Guild of Catholic Doctors, London, St Pauls, 1998, 32 pages, $£ 2.50$.}

This is a short booklet, providing advice on the use of advance directives from the perspective of the Guild of Catholic Doctors. It is the result of work of a number of members of the Guild of Catholic doctors and the Catholic Union. It is short and easy to read. The language is free from jargon and therefore accessible to a wide audience. It is a welcome contribution to the debate on the use of living wills. The authors provide their answers to 